\title{
Effects of mesenchymal stromal cell-derived extracellular vesicles on tumor growth
}

\section{Stefania Bruno ${ }^{1}$, Federica Collino ${ }^{2}$, Alessandra lavello ${ }^{2}$ and Giovanni Camussi ${ }^{2 *}$}

1 Department of Molecular Biotechnology and Health Sciences, University of Torino, Torino, Italy

${ }^{2}$ Department of Medical Sciences, University of Torino, Torino, Italy

\section{Edited by:}

Ana Maria Merino, Bellvitge

Biomedical Research Institute (IDIBELL), Spain

\section{Reviewed by:}

Mario Duran, UCLM, Spain

Rafael Ramirez Chamond, University

of Alcala, Spain

\section{*Correspondence:}

Giovanni Camussi, Department of

Medical Sciences, University of

Torino, Corso Dogliotti 14, I-10126

Torino, Italy

e-mail: giovanni.camussi@unito.it
Extracellular vesicles (EVs) are membrane vesicles, which are secreted by a variety of cells that have a relevant role in intercellular communication. EVs derived from various cell types exert different effects on target cells. Mesenchymal stromal cells (MSCs) are stem cells that are ubiquitously present in different tissues of the human body, and MSC-derived EVs take part in a wide range of biological processes. Of particular relevance is the effect of MSCs on tumor growth and progression. MSCs have opposing effects on tumor growth, being able either to favor angiogenesis and tumor initiation, or to inhibit progression of established tumors, according to the conditions. Different studies have reported that EVs from MSCs may exert either an anti- or a pro-tumor growth effect depending on tumor type and stage of development. In this review, we will discuss the data presented in the literature on EV-mediated interactions between MSCs and tumors.

Keywords: mesenchymal stem cells, extracellular vesicles, anti-tumor effect, pro-tumorigenic activity, in vivo tumor models

\section{INTRODUCTION}

Mesenchymal stromal cells (MSCs) are multipotent cells that reside in various tissues, and possess the capacity to differentiate into different mesodermal lineages (1-8). MSCs can be recruited to the site of inflammation and tissue injury/repair, as well as within the tumor environment (9-12). In this context, several studies have shown that MSCs may support tumor growth in vivo (1317), whereas others have reported an anti-tumorigenic effect for these cells (18-23). MSCs isolated from different tissues, such as human adipose tissue (24), breast (25), and palatine tonsils (26), have been shown to have the capacity to interfere with cancer cell proliferation, blocking tumor cell cycle in G0/G1 phases.

The different effects of MSCs on tumor growth depend on the tumor models, but also on the dose and time of administration of cell treatments (12). In particular, MSCs co-injected with tumor cells have been shown to support angiogenesis, thus facilitating tumor growth $(13-17,27)$. Conversely, intravenous or intra-tumor injection of MSCs in established tumors led to inhibition of tumor growth $(18-21,24)$. The exact mechanisms of these opposite effects remain unclear.

In vitro experiments have shown that cell contact between MSCs and tumor cells is not required for MSC biological activity, as the anti-proliferative effect was also observed with MSCconditioned medium $(22,28)$. This observation has led to the suggestion that paracrine/soluble factors are involved instead.

Extracellular vesicles (EVs) (exosomes and shedding microvesicles) are nano-particles secreted by various cell types, which contain protein, lipids, and genetic material, such as mRNA and miRNA. Transfer of this biological material to adjacent or distant cells may facilitate communication between different cell types. Secreted EVs express molecules that reflect the cells of origin and, in the field of regenerative medicine, EVs derived from MSCs
(MSC-EVs) have been shown to be able to mimic the therapeutic effects of the MSCs in kidney, cardiac, and brain injuries $(29,30)$. EVs released from MSCs could also be involved in the effects of MSCs on tumor growth and behavior. Several studies describing the influence of MSC-EVs on tumor growth have been reported. Similar to the case of MSCs, the released EVs can also have opposite effects on tumor growth, depending on the tumor type and the experimental animal models.

\section{STATE OF THE ART ON MSC-EV CONTENT}

Mesenchymal stromal cell-EVs express surface molecules that are characteristic of the cells origin, such as CD29, CD73, CD44, and CD105 (31). Moreover, MSC-EVs contain cytoplasmatic proteins associated with intracellular vesicle biogenesis and trafficking ( $\mathrm{RAB}$ protein family), and proteins associated with MSC self-renewal and differentiation (TGF- $\beta$, MAPK, PPAR, etc.) (32).

Mesenchymal stromal cell-EVs also contain nucleic acids (mRNA and non-coding RNA). The mRNAs present in EVs are representative of the multiple differentiation and functional properties of MSCs, including transcripts related to several different cell functions (e.g., the control of transcription, cell proliferation, and immune regulation) (33). EVs from MSCs also contain mRNA for receptors of specific growth factors, such as mRNA for the insulin growth factor 1 (IGF-1) receptor (34). MSC-EVs are able to transfer the IGF-1 receptor mRNA to target renal tubular cells in an in vitro model of renal toxic injury, inducing proliferation of proximal tubular cells (34).

EVs released by MSCs, also contain specific non-coding RNA, such as miRNAs. miRNAs are small non-coding RNAs that regulate gene expression post-transcriptionally by targeting specific mRNAs. EVs from different cell types have been shown to contain selected patterns of miRNAs $(35,36)$, which can be subsequently 
transferred to target cells $(36,37)$. The EV-shuttled miRNAs were functionally active, evident from their ability to down-regulate proteins targeted by selected transferred miRNAs (36-39).

Gene ontology analysis of the molecules targeted by the highly expressed miRNAs in MSC-derived EVs revealed genes involved in multi-organ development, cell survival, and differentiation (36).

\section{ANTI-TUMOR EFFECT OF MSC-EVs}

It has been demonstrated that MSC-EVs inhibited the proliferation of HepG2 hepatoma, Kaposi's sarcoma (KS), and Skov-3 ovarian cancer cell lines, in vitro (40). Specifically, MSC-EVs increased the percentage of tumor cells in G0/G1 phase, indicating a block in cell cycle progression. Moreover, in hepatoma and KS cancer cell lines, MSC-EV treatment induced apoptosis, as demonstrated by cytofluorimetric analyses (sub-G1 peak in cell cycle studies and activation of Caspase 8 and/or 9) and by Tunel assay. By contrast, in Skov-3 cells, EVs induced cell death by necrosis. Gene array profiles showed that, after $24 \mathrm{~h}$ of in vitro stimulation with MSC-EVs, different genes were modulated in the various cancer cell lines. In particular, the activation of negative regulators of the cell cycle (e.g., retinoblastoma 1 and retinoblastoma-like 1 and 2, etc.), and the down-regulation of genes involved in cell cycle progression (e.g., different types of cyclins) have been reported (40). These gene variations may explain the arrest of cell proliferation, which results in cell death by apoptosis or necrosis, observed in the different cancer cell lines after MSC-EV treatment (40).

To define the effect of EVs on tumor growth in vivo, HepG2, KS, and Skov-3 cells were subcutaneously injected into SCID mice. After tumors were established $\left(15 \mathrm{~mm}^{3}\right.$ in volume $)$, treatment with EVs began, by means of weekly intra-tumor injections of MSCEVs. Administration of MSC-EVs significantly inhibited tumor growth of all the tested cell lines (40).

The specificity of MSC-EVs was demonstrated by the absence of in vitro and in vivo anti-tumor effects of EVs that were derived from human dermal fibroblasts $(40,41)$.

Another recent paper described the effect of EVs derived from human cord blood Wharton's jelly MSCs (hWJMSC-EVs) on the growth of T24 bladder tumors in vitro and in vivo (41). As shown for BM-MSCs, hWJMSC-EVs also inhibited cancer cell viability by cell cycle arrest, and by induction of apoptosis, in a dosedependent fashion, both in vitro and in vivo. In this case, T24 cells were pre-stimulated with EVs prior to in vivo injection in nude mice. The anti-proliferative and pro-apoptotic effects were mediated by the down-regulation of Akt phosphorylation and the up-regulation of Caspase-3 cleavage (41).

In addition, EVs derived from human liver stem cells (HLSCs) have been demonstrated to have an anti-tumor effect. HLSCs inhibited the growth of HepG2 hepatoma, primary hepatocellular carcinoma, lymphoblastoma, and glioblastoma cells, both in vitro and in vivo (42). This study was the first to report a relevant role of miRNA, shuttled by stem cell-derived EVs, in the anti-tumor effect. Different approaches have been used to demonstrate the role of miRNAs in the anti-tumor effect of EVs. First of all, HLSC lines deprived of miRNA content by Dicer silencing were generated (42). These HLSC populations, and their derived EVs, showed a significant reduction of the anti-tumor miRNAs $\operatorname{miR} 223(43,44), \operatorname{miR} 31(45,46), \operatorname{miR} 122(47-49)$, and miR214
(50-52). EVs derived from Dicer knock-down HLSCs showed a significant reduction of anti-tumor activities, both in vitro and in vivo (42). Another approach for demonstrating the involvement of these miRNAs in the anti-tumor activity of EV-HLSCs was the use of specific miRNA inhibitors against the anti-tumor miRNAs shuttled by EV-HLSC, such as miR451, miR223, miR24, miR125b, and miR31. This strategy resulted in a reduction of the pro-apoptotic in vitro activity of EV-HLSCs on hepatoma cells (42). Moreover, the relevance of miR31 and miR451 in the anti-tumor effect of EV-HLSCs was supported by experiments showing that the correspondent miRNA mimics induced tumor regression (42).

Extracellular vesicles derived from murine MSCs were also shown to significantly down-regulate the expression of vascular endothelial growth factor (VEGF) in breast cancer cells, causing an inhibition of angiogenesis both in vitro and in vivo (53). EVs derived from MSCs were shown to shuttle anti-angiogenic molecules. Specifically, they were particularly enriched in miR16, known to target VEGF (54). Treatment with MSC-derived EVs did not affect tumor cell proliferation and viability, but downregulated the mRNA and protein levels of VEGF in tumor cells, in a dose-dependent manner (53). The transfer of miR16 from MSCs to cancer cells by means of $\mathrm{EV}$ has been indicated as the main mechanism for the anti-angiogenic effect of murine MSC-derived exosomes (53).

\section{PRO-TUMOR GROWTH EFFECT OF MSC-DERIVED EVs}

When human gastric and colon cancer cell lines (SGC-7901 and SW480, respectively) were mixed with MSCs or MSC-derived EVs, and injected subcutaneously in nude mice, an increase of tumor incidence and growth was observed (55). This effect was attributed to an enhancement of cancer cell proliferation in vivo, as shown by an increase of the proliferating cell nuclear antigen (PCNA) positive cells in tumors. In vitro, the pro-proliferative effect on cancer cells was not observed, and there were no differences in the percentage of cells in the G0/G1, S, and G2/M phases between EV-treated and untreated cells. The authors observed a dosedependent increment of VEGF and CXCR4 mRNA and protein in cancer cells at $48 \mathrm{~h}$ after incubation with exosomes. Indeed, VEGF and CXCR4 are critical for tumor growth and angiogenesis. These data suggested that EVs did not directly stimulate proliferation of cancer cells, but rather induced a pro-angiogenic program that could favor tumor engraftment and growth. This pro-angiogenic effect was confirmed in vivo, where an increment of tumor vascularization was observed. Moreover, the authors reported that EV treatment enhanced VEGF expression in cancer cells by activation of the ERK1/2 pathway. Inhibition of this pathway counteracted the increase of VEGF levels induced by EV treatment (55).

The same group that demonstrated the anti-tumor activity of hWJMSC-EVs in bladder cancer recently reported that the same EVs can in fact promote growth and aggressiveness of a renal carcinoma cell line (786-0), both in vitro and in vivo (56). In this context, after $48 \mathrm{~h}$ of incubation, EVs facilitated the cell cycle progression from G0/G1 to $S$ phase. These data were confirmed in vivo, by detection of up-regulation of cyclin D1 expression, which favors the cell cycle transition from G1 to S phase. Interestingly, pre-treatment of EVs with RNase abrogated both the in vitro 
Table 1 | EVs from different MSC source have opposite effect on different tumor types.

\begin{tabular}{lllc}
\hline Source of EVs & Tumor type & $\begin{array}{l}\text { Effect on } \\
\text { tumor growth }\end{array}$ & Reference \\
\hline Human BM-MSCs & $\begin{array}{l}\text { Hepatoma } \\
\text { Kaposi's sarcoma } \\
\text { Ovarian cancer }\end{array}$ & $\begin{array}{l}\text { Inhibition } \\
\text { Inhibition }\end{array}$ & 40 \\
& $\begin{array}{l}\text { Inhibition } \\
\text { Gastric and colon }\end{array}$ & Promotion & 55 \\
cancer & Inhibition & 53 \\
Murine BM-MSCs & Brest cancer & Inhibition & 41 \\
Cord blood & Bladder cancer & Promotion & 56 \\
Wharton's jelly MSCs & Renal cancer & Inhibition & 42 \\
HLSCs & Hepatoma & Inhibition \\
& Lymphoblast & Inhibition & \\
\hline
\end{tabular}

and in vivo effects of EVs on tumor cells, indicating the crucial involvement of the mRNAs, shuttled by EVs, in promoting proliferation in renal carcinoma cells. In addition, when renal carcinoma cells were mixed with EVs and subcutaneously injected in mice, the authors observed an up-regulation of hepatocyte growth factor (HGF) expression, at the mRNA and protein levels. hWJMSC-EVs containing HGF mRNA, with subsequent delivery of this mRNA into cancer cells, via EVs, may be one of the possible mechanisms of action. Moreover, after EV treatment, activation of the AKT and ERK1/2 pathways was observed in cancer cells, both in vitro and in vivo. To demonstrate the association of HGF induction with activation of AKT and ERK1/2, a c-Met inhibitor was added in vitro to block $\mathrm{HGF} / \mathrm{c}-\mathrm{Met}$ signaling. Under these conditions, the EV-induced activation of AKT and ERK1/2 was abrogated, with a consequent inhibition of cancer cell proliferation (56).

These results (Table 1) reveal that the same EVs can have opposite effects on different tumors, highlighting the necessity of comparative studies on different cell types in order to identify whether MSC-derived EVs exert a beneficial or a detrimental effect on the particular tumor.

\section{DIFFERENT APPROACHES FOR INCREASING THE ANTI-TUMOR EFFECT OF MSC-DERIVED EVs FOR CLINICAL APPLICATION}

Given these variable results, it is evident that, before even beginning to consider MSC-derived EVs as a potential therapeutic tool, it is necessary to define the mechanisms of their anti-tumor activity and the application context. This requires the identification of molecules with potential healing properties for a given tumor, and the development of strategies for vesicle loading and specific targeting. As EVs protect nucleic acids from degrading enzymes, one possible approach is the de novo expression, or the increased expression, of specific components (mRNA, miRNA, and siRNA) with anti-tumor activity, by genetic modification of MSCs. Overexpression of specific miRNAs in the cells of origin leads to an augmented secretion of these miRNAs in EVs (39). Recently, the possibility of using EVs, purified by MSCs, as a vehicle to delivery anti-tumor miRNA has been tested, in a rodent model of malignant glioma (57). MSCs were transfected with a plasmid encoding for miR-146, and EVs were recovered $48 \mathrm{~h}$ later. Plasmid-expressed miRNA was thus packaged into EVs. These particular EVs were tested in vivo by direct intracranial injection in rats with gliosarcoma. A single intra-tumor injection of miR-146 EVs was shown to significantly reduce tumor size (57).

The characteristic of EVs of being able to cross the bloodbrain barrier, may allow delivery of therapeutic substances to tumors of the nervous system. MSCs and brain parenchymal cells were shown to reciprocally communicate via EVs by transfer of miR-133b, ensuing modulation of neurite outgrowth (58). Specific targeting for neurons was achieved by Alvarez-Erviti et al. (59), who engineered EV-producing cells to express the protein Lamp2b fused to the neuron-specific RVG peptide, in order to obtain neuronal localization, and to deliver BACE-1 siRNA. In this way, they were able to obtain silencing of BACE1 , which is a beta secretase responsible for generation of toxic beta amyloid formation and deposition in Alzheimer's disease (59). Several other studies have indicated the possibility of ex vivo manipulation of miRNA content of EVs, for therapeutic purposes (60-62).

Moreover, EVs may deliver biologically active proteins that could influence the phenotype of recipient cells. For example, MSCs have been successfully transduced with viral vectors so that they release functional interferon alpha (IFN- $\alpha$ ), which is clinically used to treat various types of cancer (63). In this case, injection of MSC-producing IFN- $\alpha$, the derived EVs with tropism for tumors, can provide an innovative therapy for cancer treatment, by direct delivery of IFN- $\alpha$ into tumors.

\section{CONCLUSIONS}

In the contest of cancer, EVs derived from MSCs have been shown to mimic most of the beneficial and detrimental effects of the cells of origin. These opposing effects observed in different tumor types could depend on the different pathways involved. It is therefore critical to identify which molecules, shuttled by EVs, could interfere with these pathways, and therefore which kind of tumors may benefit from MSC-EV treatment. Another critical point is the timing of EV treatment. Studies based on pre-incubation of cancer cells with EVs have mainly provided information on their role in tumor engraftment and growth, but without providing a therapeutic strategy. The intra-tumor administration of EVs in an established tumor better demonstrates a therapeutic application for EVs. However, local administration may be complicated in patients, and studies are required to evaluate whether an intravenous injection of EVs is equally efficient. As in the case of MSCs, EVs may accumulate within tumors, and, therefore, could be exploited for drug delivery. Anti-tumor miRNAs and specific siRNAs are candidates for delivery by EVs. To envisage a therapeutic use of EVs, a scalable production of nonimmunogenic EVs is necessary. MSCs are potential candidates for this, but the cell senescence with the subsequent cell culture passages need to be encompassed. A possible strategy, developed by Chen et al. (64) was based on oncogenic immortalization of human embryonic stem cell-derived MSCs. These authors showed a quantitative persistent production of EVs that did not contain 
the oncogene, and maintained the same properties of EVs derived from non-immortalized cells.

In conclusion, native MSC-derived EVs have been shown to possess therapeutic potential in some but not all tumors. The strategies to engineer EVs may be exploited to deliver anti-tumor molecules, by crossing physiological barriers.

\section{ACKNOWLEDGMENTS}

Source of funding: Associazione Italiana per la Ricerca sul Cancro (AIRC), grant number: IG12890.

\section{REFERENCES}

1. Dominici M, Le Blanc K, Mueller I, Slaper-Cortenbach I, Marini F, Krause D, et al. Minimal criteria for defining multipotent mesenchymal stromal cells. The International Society for Cellular Therapy position statement. Cytotherapy (2006) 8(4):315-7. doi:10.1080/14653240600855905

2. Prockop DJ. Marrow stromal cells as stem cells for not hematopoietic tissues. Science (1997) 276:71-4. doi:10.1126/science.276.5309.71

3. Pittenger MF, Mackay AM, Beck SC, Jaiswal RK, Douglas R, Mosca JD, et al. Multilineage potential of adult human mesenchymal stem cells. Science (1999) 284:143-7. doi:10.1126/science.284.5411.143

4. Collins JJ, Thébaud B. Lung mesenchymal stromal cells in development and disease: To serve and to protect? Antioxid Redox Signal (2014). Forthcoming.

5. Tobita M, Mizuno H. Adipose-derived stem cells and periodontal tissue engineering. Int J Oral Maxillofac Implants (2013) 28:e487-93. doi:10.11607/ jomi.te29

6. Hilkens P, Gervois P, Fanton Y, Vanormelingen J, Martens W, Struys T, et al. Effect of isolation methodology on stem cell properties and multilineage differentiation potential of human dental pulp stem cells. Cell Tissue Res (2013) 353:65-78. doi:10.1007/s00441-013-1630-x

7. Feisst V, Brooks A, Chen J, Dunbar PR. Characterization of mesenchymal progenitor cell populations directly derived from human dermis. Stem Cells Dev (2014) 23(6):631-42. doi:10.1089/scd.2013.0207

8. Keating A. Mesenchymal stromal cells: new directions. Cell Stem Cell (2012) 10:709-16. doi:10.1016/j.stem.2012.05.015

9. Ji JF, He BP, Dheen ST, Tay SS. Interactions of chemokines and chemokine receptors mediate the migration of mesenchymal stem cells to the impaired site in the brain after hypoglossal nerve injury. Stem Cells (2004) 22:415-27. doi:10.1634/stemcells.22-3-415

10. Tögel F, Hu Z, Weiss K, Isaac J, Lange C, Westenfelder C. Administered mesenchymal stem cells protect against ischemic acute renal failure through differentiation-independent mechanisms. Am J Physiol Renal Physiol (2005) 289:F31-42. doi:10.1152/ajprenal.00007.2005

11. Herrera MB, Bussolati B, Bruno S, Morando L, Mauriello-Romanazzi G, Sanavio F, et al. Exogenous mesenchymal stem cells localize to the kidney by means of CD44 following acute tubular injury. Kidney Int (2007) 72(4):430-41. doi:10.1038/sj.ki.5002334

12. Klopp AH, Gupta A, Spaeth E, Andreeff M, Marini FIII. Concise review: dissecting a discrepancy in the literature: do mesenchymal stem cells support or suppress tumor growth? Stem Cells (2011) 29:11-9. doi:10.1002/stem.559

13. Zhu W, Xu W, Jiang R, Qian H, Chen M, Hu J, et al. Mesenchymal stem cells derived from bone marrow favour tumor cell growth in vivo. Exp Mol Pathol (2006) 80(3):267-74. doi:10.1016/j.yexmp.2005.07.004

14. Yu JM, Jun ES, Bae YC, Jung JS. Mesenchymal stem cells derived from human adipose tissues favor tumor cell growth in vivo. Stem Cells Dev (2008) 17:463-73. doi:10.1089/scd.2007.0181

15. Djouad F, Bony C, Apparailly F, Louis-Plence P, Jorgensen C, Noel D. Earlier onset of syngenic tumors in the presence of mesenchymal stem cells. Transplantation (2006) 82(8):1060-6. doi:10.1097/01.tp.0000236098.13804.0b

16. Ramasamy R, Lam EW, Soeiro I, Tisato V, Bonnet D, Dazzi F. Mesenchymal stem cells inhibit proliferation and apoptosis of tumor cells: impact on in vivo tumor growth. Leukemia (2007) 21:304-10. doi:10.1038/sj.leu.2404489

17. Wong RS. Mesenchymal stem cells: angels or demons? J Biomed Biotechnol (2011) 2011:459510. doi:10.1155/2011/459510

18. Khakoo AY, Pati S, Anderson SA, Reid W, Elshal MF, Rovira II, et al. Human mesenchymal stem cells exert potent anti-tumorigenic effects in a model of Kaposi's sarcoma. J Exp Med (2006) 203(5):1235-47. doi:10.1084/jem.20051921
19. Qiao L, Xu Z, Zhao T, Zhao Z, Shi M, Zhao RC, et al. Suppression of tumorigenesis by human mesenchymal stem cells in a hepatoma model. Cell Res (2008) 18(4):500-7. doi:10.1038/cr.2008.40

20. Secchiero P, Zorzet S, Tripodo C, Corallini F, Melloni E, Caruso L, et al. Human bone marrow mesenchymal stem cells display anti-cancer activity in SCID mice bearing disseminated Non-Hodgkin's lymphoma xenografts. PLoS One (2010) 6(6):e11140. doi:10.1371/journal.pone.0011140

21. Li GC, Ye QH, Xue YH, Sun HJ, Zhou HJ, Ren N, et al. Human mesenchymal stem cells inhibit metastasis of a hepatocellular carcinoma model using the MHCC97-H cell line. Cancer Sci (2010) 101(12):2546-53. doi:10.1111/j.13497006.2010.01738.x

22. Li L, Tian H, Chen Z, Yue W, Li S, Li W. Inhibition of lung cancer cell proliferation mediated by human mesenchymal stem cells. Acta Biochim Biophys Sin (2011) 43:143-8. doi:10.1093/abbs/gmq118

23. Otsu K, Das S, Houser SD, Quadri SK, Bhattacharya S, Bhattacharya J. Concentration-dependent inhibition of angiogenesis by mesenchymal stem cells. Blood (2009) 113:4197-205. doi:10.1182/blood-2008-09-176198

24. Cousin B, Ravet E, Poglio S, De Toni F, Bertuzzi M, Lulka H, et al. Stromal cells derived from human adipose tissue provoke pancreatic cancer cell death both in vitro and in vivo. PLoS One (2009) 4(7):e6278. doi:10.1371/journal.pone. 0006278

25. Dong-Le Bourhis X, Berthois Y, Millot G, Degeorges A, Sylvi M, Martin PM, et al. Effect of stromal and epithelial cells derived from normal and tumorous breast tissue on the proliferation of human breast cancer cell lines in co-culture. Int J Cancer (1997) 71(1):42-8. doi:10.1002/(SICI)1097-0215(19970328)71:1<42: :AID-IJC9>3.0.CO;2-3

26. Lim YS, Lee JC, Lee YS, Lee BJ, Wang SG. Growth inhibitory effect of palatine tonsil-derived mesenchymal stem cells on head and neck squamous cell carcinoma cells. Clin Exp Otorhinolaryngol (2012) 5(2):86-93. doi:10.3342/ceo.2012. 5.2 .86

27. Kucerova L, Matuskova M, Hlubinova K, Altanerova V, Altaner C. Tumor cell behavior modulation by mesenchymal stromal cells. Mol Cancer (2010) 9:129. doi:10.1186/1476-4598-9-129

28. Tian LLH, Yue W, Zhu F, Li S, Li W. Human mesenchymal stem cells play a dual role on tumor cell growth in vitro and in vivo. J Cell Physiol (2011) 226:1860-7. doi:10.1002/jcp.22511

29. Bruno S, Camussi G. Role of mesenchymal stem cell-derived microvesicles in tissue repair. Pediatr Nephrol (2013) 28(12):2249-54. doi:10.1007/s00467-0132413-z

30. Katsuda T, Kosaka N, Takeshita F, Ochiya T. The therapeutic potential of mesenchymal stem cell-derived extracellular vesicles. Proteomics (2013) 13(1011):1637-53. doi:10.1002/pmic.201200373

31. Camussi G, Deregibus MC, Bruno S, Cantaluppi V, Biancone L. Exosomes/microvesicles as a mechanism of cell-to-cell communication. Kidney Int (2010) 789:838-48. doi:10.1038/ki.2010.278

32. Kim HS, Choi DY, Yun SJ, Choi SM, Kang JW, Jung JW, et al. Proteomic analysis of microvesicles derived from human mesenchymal stem cells. J Proteome Res (2012) 11:839-49. doi:10.1021/pr200682z

33. Bruno S, Grange C, Deregibus MC, Calogero RA, Saviozzi S, Collino F, et al. Mesenchymal stem cell-derived microvesicles protect against acute tubular injury. J Am Soc Nephrol (2009) 20:1053-67. doi:10.1681/ASN.2008070798

34. Tomasoni S, Longaretti L, Rota C, Morigi M, Conti S, Gotti E, et al. Transfer of growth factor receptor mRNA via exosomes unravels the regenerative effect of mesenchymal stem cells. Stem Cells Dev (2013) 22:772-80. doi:10.1089/scd.2012.0266

35. Valadi H, Ekström K, Bossios A, Sjöstrand M, Lee JJ, Lötvall JO. Exosome-mediated transfer of mRNAs and microRNAs is a novel mechanism of genetic exchange between cells. Nat Cell Biol (2007) 9(6):654-9. doi:10.1038/ncb1596

36. Collino F, Deregibus MC, Bruno S, Sterpone L, Aghemo G, Viltono L, et al. Microvesicles derived from adult human bone marrow and tissue specific mesenchymal stem cells shuttle selected pattern of miRNAs. PLoS One (2010) 5(7):e11803. doi:10.1371/journal.pone.0011803

37. Yuan A, Farber EL, Rapoport AL, Tejada D, Deniskin R, Akhmedov NB, et al. Transfer of microRNAs by embryonic stem cell microvesicles. PLoS One (2009) 4:4722. doi:10.1371/journal.pone.0004722

38. Zhang Y, Liu D, Chen X, Li J, Li L, Bian Z, et al. Secreted monocytic miR-150 enhances targeted endothelial cell migration. Mol Cell (2010) 39(1):133-44. doi:10.1016/j.molcel.2010.06.010 
39. Kosaka N, Iguchi H, Yoshioka Y, Takeshita F, Matsuki Y, Ochiya T. Secretory mechanisms and intercellular transfer of microRNAs in living cells. J Biol Chem (2010) 285(23):17442-52. doi:10.1074/jbc.M110.107821

40. Bruno S, Collino F, Deregibus MC, Grange C, Tetta C, Camussi G. Microvesicles derived from human bone marrow mesenchymal stem cells inhibit tumor growth. Stem Cells Dev (2013) 22(5):758-71. doi:10.1089/scd.2012.0304

41. Wu S, Ju GQ, Du T, Zhu YJ, Liu GH. Microvesicles derived from human umbilical cord Wharton's jelly mesenchymal stem cells attenuate bladder tumor cell growth in vitro and in vivo. PLoS One (2013) 8(4):e61366. doi:10.1371/journal. pone.0061366

42. Fonsato V, Collino F, Herrera MB, Cavallari C, Deregibus MC, Cisterna B, et al. Human liver stem cell-derived microvesicles inhibit hepatoma growth in SCID mice by delivering antitumor microRNAs. Stem Cells (2012) 30(9):1985-98. doi:10.1002/stem.1161

43. Wu L, Li H, Jia CY, Cheng W, Yu M, Peng M, et al. MicroRNA-223 regulates FOXO1 expression and cell proliferation. FEBS Lett (2012) 586(7):1038-43. doi:10.1016/j.febslet.2012.02.050

44. Dong YW, Wang R, Cai QQ, Qi B, Wu W, Zhang YH, et al. Sulfatide epigenetically regulates miR-223 and promotes the migration of human hepatocellular carcinoma cells. J Hepatol (2014) 60(4):792-801. doi:10.1016/j.jhep.2013.12.004

45. Creighton CJ, Fountain MD, Yu Z, Nagaraja AK, Zhu H, Khan M, et al. Molecular profiling uncovers a p53-associated role for microRNA-31 in inhibiting the proliferation of serous ovarian carcinomas and other cancers. Cancer Res (2010) 70:1906-15. doi:10.1158/0008-5472.CAN-09-3875

46. Karnuth B, Dedy N, Spieker T, Lawlor ER, Gattenlöhner S, Ranft A, et al. Differentially expressed miRNAs in Ewing sarcoma compared to mesenchymal stem cells: low miR-31 expression with effects on proliferation and invasion. PLoS One (2014) 9(3):e93067. doi:10.1371/journal.pone.0093067

47. Tsai WC, Hsu PW, Lai TC, Chau GY, Lin CW, Chen CM, et al. MicroRNA-122, a tumor suppressor microRNA that regulates intrahepatic metastasis of hepatocellular carcinoma. Hepatology (2009) 49(5):1571-82. doi:10.1002/hep.22806

48. Yin J, Tang HF, Xiang Q, Yu J, Yang XY, Hu N, et al. MiR-122 increases sensitivity of drug-resistant BEL-7402/5-FU cells to 5-fluorouracil via down-regulation of bcl-2 family proteins. Pharmazie (2011) 66:975-81.

49. Wang G, Dong X, Tian W, Lu Y, Hu J, Liu Y, et al. Evaluation of miR-122regulated suicide gene therapy for hepatocellular carcinoma in an orthotopic mouse model. Chin J Cancer Res (2013) 25(6):646-55. doi:10.3978/j.issn.10009604.2013.11.07

50. Duan Q, Wang X, Gong W, Ni L, Chen C, He X, et al. ER stress negatively modulates the expression of the miR-199a/214 cluster to regulates tumor survival and progression in human hepatocellular cancer. PLoS One (2012) 7:e31518. doi:10.1371/journal.pone.0031518

51. Lu S, Gao Y, Huang X, Wang X. Cantharidin exerts anti-hepatocellular carcinoma by miR-214 modulating macrophage polarization. Int J Biol Sci (2014) 10(4):415-25. doi:10.7150/ijbs.8002

52. Chen DL, Wang ZQ, Zeng ZL, Wu WJ, Zhang DS, Luo HY, et al. Identification of miR-214 as a negative regulator of colorectal cancer liver metastasis via regulation of FGFR1 expression. Hepatology (2014) 60(2):598-609. doi:10.1002/hep. 27118

53. Lee JK, Park SR, Jung BK, Jeon YK, Lee YS, Kim MK, et al. Exosomes derived from mesenchymal stem cells suppress angiogenesis by down-regulating VEGF expression in breast cancer cells. PLoS One (2013) 12:e84256. doi:10.1371/ journal.pone.0084256
54. Hua Z, Lv Q, Ye W, Wong CK, Cai G, Gu D, et al. MiRNA-directed regulation of VEGF and other angiogenic factors under hypoxia. PLoS One (2006) 27(1):e116. doi:10.1371/journal.pone.0000116

55. Zhu W, Huang L, Li Y, Zhang X, Gu J, Yan Y, et al. Exosomes derived from human bone marrow mesenchymal stem cells promote tumor growth in vivo. Cancer Lett (2012) 315(1):28-37. doi:10.1016/j.canlet.2011.10.002

56. Du T, Ju G, Wu S, Cheng Z, Cheng J, Zou X, et al. Microvesicles derived from human Wharton's jelly mesenchymal stem cells promote human renal cancer cell growth and aggressiveness through induction of hepatocyte growth factor. PLoS One (2014) 9(5):e96836. doi:10.1371/journal.pone.0096836

57. Katakowski M, Buller B, Zheng X, Lu Y, Rogers T, Osobamiro O, et al. Exosomes from marrow stromal cells expressing miR-146b inhibit glioma growth. Cancer Lett (2013) 335(1):201-4. doi:10.1016/j.canlet.2013.02.019

58. Xin H, Li Y, Buller B, Katakowski M, Zhang Y, Wang X, et al. Exosomemediated transfer of miR-133b from multipotent mesenchymal stromal cells to neural cells contributes to neurite outgrowth. Stem Cells (2012) 30(7):1556-64. doi:10.1002/stem.1129

59. Alvarez-Erviti L, Seow Y, Yin H, Betts C, Lakhal S, Wood MJ. Delivery of siRNA to the mouse brain by systemic injection of targeted exosomes. Nat Biotechnol (2011) 29(4):341-5. doi:10.1038/nbt.1807

60. Akao Y, Iio A, Itoh T, Noguchi S, Itoh Y, Ohtsuki Y, et al. Microvesicle-mediated RNA molecule delivery system using monocytes/macrophages. Mol Ther (2011) 19(2):395-9. doi:10.1038/mt.2010.254

61. van den Boorn JG, Schlee M, Coch C, Hartmann G. SiRNA delivery with exosome nanoparticles. Nat Biotechnol (2011) 29(4):325-6. doi:10.1038/nbt.1830

62. El Andaloussi S, Lakhal S, Mäger I, Wood MJ. Exosomes for targeted siRNA delivery across biological barriers. Adv Drug Deliv Rev (2013) 65(3):391-7. doi:10.1016/j.addr.2012.08.008

63. Xu C, Lin L, Cao G, Chen Q, Shou P, Huang Y, et al. Interferon- $\alpha$-secreting mesenchymal stem cells exert potent antitumor effect in vivo. Oncogene (2013). doi:10.1038/onc.2013.458

64. Chen TS, Arslan F, Yin Y, Tan SS, Lai RC, Choo AB, et al. Enabling a robust scalable manufacturing process for therapeutic exosomes through oncogenic immortalization of human ESC-derived MSCs. J Transl Med (2011) 25(9):47. doi:10.1186/1479-5876-9-47

Conflict of Interest Statement: The authors declare that the research was conducted in the absence of any commercial or financial relationships that could be construed as a potential conflict of interest.

Received: 05 June 2014; paper pending published: 08 July 2014; accepted: 25 July 2014; published online: 11 August 2014.

Citation: Bruno S, Collino F, Iavello A and Camussi G (2014) Effects of mesenchymal stromal cell-derived extracellular vesicles on tumor growth. Front. Immunol. 5:382. doi: 10.3389/fimmu.2014.00382

This article was submitted to Immunotherapies and Vaccines, a section of the journal Frontiers in Immunology.

Copyright (c) 2014 Bruno, Collino, Iavello and Camussi. This is an open-access article distributed under the terms of the Creative Commons Attribution License (CC BY). The use, distribution or reproduction in other forums is permitted, provided the original author(s) or licensor are credited and that the original publication in this journal is cited, in accordance with accepted academic practice. No use, distribution or reproduction is permitted which does not comply with these terms. 